\title{
Associations between nailfold capillaroscopy findings and interstitial lung disease in patients with mixed connective tissue disease
}

\author{
Magdalena Celińska-Löwenhoff ${ }^{1}$, Maciej Pastuszczak², Karolina Pełka², Magdalena Stec-Polak², \\ Anna Wojas-Pelc², Jacek Musiał ${ }^{1}$
}

\begin{abstract}
${ }^{1} 2^{\text {nd }}$ Department of Medicine, Jagiellonian University Medical College, Krakow, Poland ${ }^{2}$ Department of Dermatology, Jagiellonian University Medical College, Krakow, Poland
\end{abstract}

Submitted: 27 September 2018

Accepted: 25 October 2018

Arch Med Sci 2020; 16 (2): 297-301

DOI: https://doi.org/10.5114/aoms.2018.81129

Copyright (C) 2019 Termedia \& Banach

\section{Abstract}

Introduction: Mixed connective tissue disease (MCTD) is a chronic immune-mediated disorder defined by the combined presence of serum antiRNP antibodies and distinct clinical features including progressive lung fibrosis. The aim of this study was to evaluate potential associations between lung fibrosis in MCTD and specific clinical and laboratory findings including results of nailfold capillaroscopy (NFC) examination.

Material and methods: Patients with MCTD who were admitted to the Departments of Allergy and Immunology or Dermatology at the University Hospital of Krakow (Poland) in 2015-2018 were identified based on comprehensive individual record review. Diagnosis of MCTD required fulfilment of at least one of the four widely accepted sets of diagnostic criteria. Clinical and laboratory data were collected, and statistical analysis was performed to identify potential predictors of interstitial lung disease (ILD).

Results: Thirty patients (90\% females) aged 22-81 years met the study inclusion criteria. The mean duration of symptoms associated with MCTD was 7.3 months. Photosensitivity and Raynaud's phenomenon were the most common clinical manifestations ( $90 \%$ and $70 \%$, respectively). Hand oedema, sclerodactyly and the presence of giant capillaries in NFC correlated significantly with the risk of lung involvement. In multivariate analysis, the presence of enlarged loops in NFC (giant capillaries) was identified as an independent factor for ILD $\left(R^{2}=0.82, p<0.0000001\right)$.

Conclusions: The NFC examination should be considered in all patients with a diagnosis of MCTD. The presence of giant capillaries may be a promising marker for interstitial lung disease in these patients, especially among those with a short duration of disease (i.e. $<1$ year).

Key words: capillaroscopy, mixed connective tissue disease, interstitial lung disease.

\section{Introduction}

Mixed connective tissue disease (MCTD) is a systemic autoimmune disease characterized by the presence of autoantibodies targeting the U1-RNP and the simultaneous presence of distinct clinical features (e.g. myositis, lung fibrosis, skin lesions) [1]. Morbidity and mortality in MCTD are driven mainly by lung involvement. A recent systematic evaluation of lung involvement among MCTD patients revealed at least one high res-

\author{
Corresponding author: \\ Maciej Pastuszczak MD, PhD \\ Department of Dermatology \\ Jagiellonian University \\ Medical College \\ 8 Skawińska St \\ 31-066 Krakow, Poland \\ Phone: +48 124305266 \\ ext. 7416 \\ E-mail: mpastuszczak@wp.pl
}


olution computed tomography (HRCT) abnormality compatible with interstitial lung disease (ILD) in $52 \%$ of included individuals [2]. In a separate study, severe lung fibrosis was found in 19\% of patients with MCTD [3]. Moreover, patients with MCTD and severe lung fibrosis had almost seven times higher risk of death compared with those with normal HRCT scanning [2]. Recent studies showed that if left untreated, ILD in patients with MCTD progresses. However, progression of lung disease does not seem to be as rapid as can occur in systemic sclerosis or dermatomyositis/polymyositis [3].

Theoretically, early detection of lung involvement and more aggressive treatment protocols may improve outcomes in patients with MCTD. Previous work has suggested that anti-Ro52 antibodies may serve as a potential marker for lung fibrosis in MCTD [4]. Nailfold capillaroscopy (NFC) is a non-invasive diagnostic tool for patients with different connective tissue diseases (CTDs) that permits the detection of local microvascular changes that correlate with systemic vascular involvement. Interestingly, structural abnormalities in the capillary bed, found in NFC, very often precede the onset of systemic manifestations of CTDs. Therefore, the associations between HRCT abnormalities and some clinical features and results of nailfold capillaroscopy (NFC) were investigated in a well-defined MCTD cohort characterized by short disease duration (i.e. $<1$ year).

\section{Material and methods}

\section{Study cohort}

Hospital records from 179 patients who had attended the Departments of Allergy and Immunology or Dermatology at the University Hospital of Cracow (Poland) from 2015 to 2018 were reviewed to identify those diagnosed with MCTD (i.e. M35.1) by the ICD-10. Study inclusion criteria comprised: age $\geq 18$ years, clinical diagnosis of MCTD, positive anti-RNP test, fulfilment of at least one of the criteria set for MCTD (i.e. the criteria of Sharp [5], Alarcon-Segovia and Villareal [6], Kasukawa [7], or Kahn and Appelboom [8]), exclusion of other connective tissue diseases and a disease duration of no longer than one year. Additionally, only patients with complete HRCT scan data, laboratory results (complete blood count), autoantibody analyses and NFC at disease onset were included. Altogether, 30 patients met the inclusion criteria and were enrolled in this study.

\section{Autoantibody analysis}

Autoantibodies directed at various cellular compartments (including nuclear constituents, components of the nuclear envelope, mitotic spindle apparatus, cytosol, cytoplasmic organelles and cell membranes) were tested by indirect immunofluorescence (IIF) in the IgG isotype (HEp-20-10 cells and the conjugate, Euroimmun, Lübeck, Germany). Further identification of specific antibodies was performed by immunoblot (ANA Profile 3 Euroline Blot test kit; Euroimmun, Lübeck, Germany) according to the manufacturer's instructions. With this test fifteen autoantibodies have been determined, i.e. antibodies against RNP, Sm, SS-A, Ro-52, SS-B, Scl-70, PM-Scl, Jo-1, centromere protein B, PCNA, dsDNA, nucleosomes, histones, ribosomal P-proteins, AMA M2.

\section{HRCT lung scans analysis}

The presence, extent and distribution of groundglass attenuations, airspace consolidation, reticular patterns and interlobular septal thickening were evaluated according to the radiologic criteria of interstitial lung disease (ILD) recommended by the Nomenclature Committee of the Fleischner Society [9]. The extent of reticular patterns (i.e. the coarseness of the lung fibrosis) was assessed as the percentage of lung parenchyma involved with $1-14 \%$ being mild fibrosis, 15-49\% moderate fibrosis, and > 50\% severe fibrosis.

\section{Pulmonary function tests}

Pulmonary function tests (PFTs) performed in all MCTD patients included dynamic spirometry and carbon monoxide diffusion capacity. In addition, all patients underwent a 6-min walk test (6MWT).

\section{Nailfold capillaroscopy (NFC)}

In all patients an NFC was performed before inclusion in the study, as described previously [10]. Briefly, field capillary microscopy with 100x magnification was used. Eight digits (excluding thumbs) were examined using a bifocal stereomicroscope. Immersion oil was applied to increase skin transparency and the whole nailfold region including the edges was examined. Abnormal findings were defined as enlargement of capillary loops (giant capillaries; absolute number $>3$ ), presence of bushy capillaries ( $>1$ ) and avascular areas (loss of two consecutive loops of nail bed).

\section{Statistical analysis}

Statistical analysis was carried out using Statistical 7.1 PL software (TIBCO Software Inc., Palo Alto, CA, USA). If not stated otherwise, data were expressed as median and minimum-maximum values. Continuous variables were compared with the Mann-Whitney $U$ test. The $\chi^{2}$ test or Fisher's exact test was used for dichotomous variables. To identify independent factors, a multivariable logis- 
tic regression analysis was used. A $p$-value $<0.05$ was considered statistically significant.

\section{Results}

Thirty patients (90\% female) aged 22-81 years with MCTD were included in this study. Only two of them were $\geq 65$ years. All patients fulfilled at least one of the MCTD criteria sets with $53 \%$ meeting the Sharp criteria, 47\% the Alarcon-Segovia criteria, $90 \%$ the Kasukawa criteria, and $83 \%$ the Kahn criteria. The mean disease duration at the time of the study was $7.3 \pm 2$ months. The most frequent symptoms at disease onset were photosensitivity (90\%) and Raynaud's phenomenon (70\%). Interstitial lung fibrosis was identified in $6(20 \%)$ individuals and was defined as mild in all cases. None of the included patients had respiratory symptoms such as breathlessness, chest discomfort or dry cough. The patients with fibrosis had non-significantly lower PFT values and 6MWT, and a higher WHO class than the individuals with normal HRCT. Clinical and laboratory characteristics for the MCTD patients with and without interstitial lung disease are presented in Table I.

Mild leukopenia (i.e. < 4000/ $\mu \mathrm{l}$ and > 2500/ $\mu$ l) was the most common (33.3\%) complete blood count finding. Besides the obligatory presence of anti-RNP autoantibodies, the most common autoantibodies were anti-SSA (43.3\%) and anti-dsDNA (33.3\%) (Table I). Anti-Ro52 antibodies were detected only in 1 (3.33\%) patient. Antiphospholipid antibodies were not assessed routinely in our group of patients as no one had a history of thrombotic or obstetrical complications.

Almost one-fifth of patients had giant capillaries confirmed in NFC.

Hand oedema, sclerodactyly and the presence of giant capillaries in NFC correlated significantly with the risk of interstitial lung disease (Table II). No such correlation was observed for anti-Ro52 antibodies (data not shown). In a multivariate analysis, giant capillaries were identified to independently correlate with pulmonary fibrosis $\left(R^{2}=\right.$ $0.82, p<0.0000001$ ).

Of the 30 patients, 16 did not require any treatment. The remaining patients most commonly took methotrexate and hydroxychloroquine ( $23.3 \%$ and $20 \%$, respectively). Of the six individuals with lung fibrosis, four received therapy (cyclophosphamide in three cases and azathioprine in 1 case).

\section{Discussion}

Interstitial lung disease (ILD) in MCTD may affect as many as $50 \%$ of patients $[2,3]$. It impairs pulmonary function and overall physical capacity leading to increased morbidity and mor- tality. The HRCT is a valuable diagnostic tool for detection of lung fibrosis. A non-invasive marker predictive of lung involvement would be useful as an early warning of possible pulmonary fibrosis. Such a test would also help to calculate pre-test probability as an indication to perform HRCT. In consequence, it may help to start an appropriate treatment in patients who are more likely to benefit from earlier initiation of immunosuppressive therapy.

Our data indicate that identification of giant capillaries on NFC might be a promising marker heralding early lung involvement in otherwise asymptomatic patients. NFC is an easy-to-perform non-invasive test. Previously, some distinct features of microvascular pathologic alteration in NFC were found to be associated with a particular connective tissue disease [11]. Moreover, investigation of clinical-capillaroscopic alterations in patients with systemic sclerosis revealed an association with end-organ involvement (including lung disease) and rate of disease progression [12].

To the best of our knowledge, only one study has examined in detail the relationship between NFC findings in MCTD patients and internal organ involvement [13]. This study found avascular areas and reduced capillary density in NFC to be correlated significantly with interstitial lung fibrosis in patients who had MCTD for a mean of 8.5 years. Moreover, during a 3-year follow-up, the dynamic nature of capillary changes in MCTD was shown. In some patients, immunosuppressive treatment presumably resulted in normalization of reduced capillary density and in improvement of avascular areas that was associated with stabilization or improvement of lung disease.

In this study, only patients with MCTD of short disease duration (i.e. < 1 year) were enrolled. None of the studied individuals had avascular areas in NFC examination and HRCT scanning revealed only abnormalities classified as mild fibrosis. Therefore, we can hypothesize that the association between giant capillaries in NFC and lung fibrosis in our study may represent early endothelial injury, a primary target in this disease. Reduced capillary density and avascular areas may represent the next stage of disease progression. This could explain the correlation between NFC findings and more advanced lung disease. Further studies are needed to confirm these assumptions.

Further studies are also needed to detect other potential markers of disease progression including lung involvement. For example, it might be interesting to assess regulatory T-cells CD4+CD25FoxP3 (Tregs) that are associated with disease activity and organ damage in systemic lupus erythematosus (SLE) [14]. 
Table I. Clinical and laboratory characteristics of patients with mixed connective tissue disease (MCTD) with and without interstitial lung disease (ILD)

\begin{tabular}{|c|c|c|c|}
\hline Parameter & $\begin{array}{l}\text { MCTD with ILD } \\
(n=6)\end{array}$ & $\begin{array}{l}\text { MCTD without ILD } \\
(n=24)\end{array}$ & $P$-value \\
\hline Age (min.-max.) [years] & $52.5(45-62)$ & $46.5(22-81)$ & NS \\
\hline Females & $6(100)$ & $21(87.5)$ & NS \\
\hline Photosensitivity & $5(83.3)$ & $23(95.8)$ & NS \\
\hline Xerophthalmia or xerostomia & $0(0)$ & $4(16.7)$ & NS \\
\hline Muscle pain & $3(50)$ & $11(45.8)$ & NS \\
\hline Myositis & $3(50)$ & $9(37.5)$ & NS \\
\hline Fatigue & $2(33.3)$ & $10(41.7)$ & NS \\
\hline Raynaud's syndrome & $5(83.3)$ & $16(66.7)$ & NS \\
\hline Arthralgia & $4(66.7)$ & $15(62.5)$ & NS \\
\hline Arthritis & $2(33.3)$ & $6(25)$ & NS \\
\hline Oral aphthosis & $0(0)$ & $1(4.2)$ & NS \\
\hline Sclerodactyly & $4(66.6)$ & $5(20.8)$ & 0.04 \\
\hline Hand oedema & $5(83.3)$ & $5(20.8)$ & 0.01 \\
\hline Facial erythema & $2(33.3)$ & $12(50)$ & NS \\
\hline Dysphagia & $0(0)$ & $1(4.2)$ & NS \\
\hline Livedo reticularis & $0(0)$ & $5(20.8)$ & NS \\
\hline Alopecia & $0(0)$ & $4(16.7)$ & NS \\
\hline Leukopenia $(<4000 / \mu l)$ & $3(50)$ & $7(29.2)$ & NS \\
\hline Lymphopenia $(<1000 / \mu \mathrm{l})$ & $2(33.3)$ & $3(12.5)$ & NS \\
\hline Thrombocytopenia $(<150000 / \mu l)$ & $0(0)$ & $2(8.3)$ & NS \\
\hline Positive ANA & $6(100)$ & $24(100)$ & NS \\
\hline ANA nuclear homogeneous pattern & $4(66.7)$ & $3(12.5)$ & NS \\
\hline ANA nuclear speckled pattern & $6(100)$ & $24(100)$ & NS \\
\hline ANA nuclear centromere pattern & $0(0)$ & $0(0)$ & NS \\
\hline ANA nucleolar pattern & $0(0)$ & $1(4.2)$ & NS \\
\hline ANA cytoplasmic pattern & $3(50)$ & $8(33.3)$ & NS \\
\hline Anti-ds-DNA & $4(66.7)$ & $6(25)$ & NS \\
\hline Anti-SSA & $3(50)$ & $10(41.7)$ & NS \\
\hline Anti-SSB & $0(0)$ & $1(4.2)$ & NS \\
\hline Anti-Sm & $0(0)$ & $2(8.3)$ & NS \\
\hline Avascular areas in NFC & $0(0)$ & $0(0)$ & NS \\
\hline Giant capillaries in NFC & $5(83.3)$ & $0(0)$ & 0.0001 \\
\hline
\end{tabular}

Variables are shown as number (percentage) or otherwise stated. ANA - antinuclear antibodies, NFC - nailfold capillaroscopy.

Table II. Predictors of pulmonary fibrosis

\begin{tabular}{|lcc|}
\hline Variable & OR $(95 \% \mathrm{CI})$ & $P$-value \\
\hline Hand oedema & $19(1.8-202)$ & 0.009 \\
\hline Sclerodactyly & $34.5(2.4-506.1)$ & 0.01 \\
\hline Giant capillaries in NFC & $179.7(6.4-5031)$ & 0.0001 \\
\hline
\end{tabular}

$O R$ - odds ratio, NFC - nailfold capillaroscopy.
Our study has several limitations. First, due to a small sample size and enrolment of patients with relatively short disease duration (i.e. < 1 year), our results might not be generalizable to all patients with MCTD, which comprises a broad clinical spectrum. Second, the study design was retrospective and lacked longitudinal follow-up, and thus did not allow investigation of the cumu- 
lative incidence of each clinical manifestation and their progression. Third, the age range of our patients was large (22-81 years). One may note that aging patients are more prone to autoimmune reactions. The mechanism and pathophysiology of autoimmunity seem to be different in this group of patients as in younger ones. However, only two of the included patients were at the age of $\geq 65$ years, the median age was 48 years, and the group was otherwise clinically homogeneous. Finally, due to the lack of quantification of capillary alterations and scoring systems, NFC remains an operator-dependent examination.

In conclusion, this study points to an important role for NFC in the detection of lung involvement in patients with MCTD. NFC should be performed in all patients with MCTD. The enlarged capillaries in NFC may serve as an early marker of lung fibrosis in a subgroup of individuals with a diagnosis of MCTD of less than 1 year's duration. These findings need confirmation in other studies, preferably in patients with different durations of disease and various stages of interstitial lung disease.

\section{Conflict of interest}

The authors declare no conflict of interest.

\section{References}

1. Gunnarsson R, Hetlevik SO, Lilleby V, Molberg O. Mixed connective tissue disease. Best Pract Res Clin Rheum 2016; 30: 95-111.

2. Gunnarsson R, Aalokken TM, Molberg O, et al. Prevalence and severity of interstitial lung disease in mixed connective tissue disease: nationwide, cross-sectional study. Ann Rheum Dis 2012; 71: 1966-72.

3. Kawano-Dourado L, Baldi BG, Kay FU, et al. Pulmonary involvement in long-term mixed connective tissue disease: functional trends and image findings after 10 years. Clin Exp Rheumatol 2015; 33: 234-40.

4. Gunnarsson R, El-Hage F, Aalokken TM, et al. Associations between anti-Ro52 antibodies and lung fibrosis in mixed connective tissue disease. Rheumatology (Oxford) 2016; 64: 303-10.

5. Sharp GC. Diagnostic criteria for classification of MCTD. In: Mixed Connective Tissue Disease and Anti-Nuclear Antibodies. Kasukawa R, Sharp GC (eds). Elsevier Science Publishers B.V. (Biomedical Division), Amsterdam 1987; 23-30.

6. Alarcon-Segovia D, Villareal M. Classification and diagnostic criteria for mixed connective tissue disease. In: Mixed Connective Tissue Disease and Anti-Nuclear Antibodies. Kasukawa R, Sharp GC (eds). Elsevier Science Publishers B.V. (Biomedical Division), Amsterdam 1987; 33-40.

7. Kasukawa R, Tojo T, Miyawaki S. Preliminary diagnostic criteria for classification of mixed connective tissue disease. In: Mixed Connective Tissue Disease and Anti-Nuclear Antibodies. Kasukawa R, Sharp GC (eds). Elsevier Science Publishers B.V. (Biomedical Division), Amsterdam 1987; 41-7.
8. Kahn MF, Appelboom T. Syndrome de sharp. In: Les Maladies Systemiques. Kahn MF, Peltier AP, Mayer O, et al. (eds.). Flammarion, Paris 1991; 545-56.

9. Hansell DM, Bankier AA, MacMahon H, McLoud TC, Muller NL, Remy J. Fleischner Society: glossary of terms for thoracic imaging. Radiology 2008; 246: 697-722.

10. Maricq HR. Wide field capillary microscopy. Technique and rating scale for abnormalities seen in scleroderma and related disorders. Arthritis Rheum 1981; 24: 1159-65.

11. Cutolo M, Smith V. State of the art of nailfold capillaroscopy: a reliable diagnostic tool and putative biomarker in rheumatology? Rheumatology 2013; 52: 1933-40.

12. Soulaidopoulos S, Traintafyllidou E, Garyfallos A, Kitas GD, Dimitroulas T. The role of nailfold capillaroscopy in the assessment of internal organ involvement in systemic sclerosis: a critical review. Autoimm Rev 2017; 16: 787-95.

13. De Holanda Mafaldo Diogenes A, Bonfa E, Fuller R, Correia Caleiro MT. Capillaroscopy is a dynamic process in mixed connective tissue disease. Lupus 2007; 16: 254-8.

14. El-Maraghy N, Ghaly MS, Dessouki O, Nasef SI, Metwally L. CD4+CD25-FoxP3 T cells as a marker of disease activity and organ damage in systemic lupus erythematosus patients. Arch Med Sci 2018; 14: 1033-40. 